J. Phys. IV France 138 (2006) 127-140

(C) EDP Sciences, Les Ulis

DOI: 10.1051/jp4:2006138016

\title{
Potentialités des lasers à fibre dans la génération de rayonnement cohérent UV
}

\author{
G. Martel ${ }^{1}$, A. Hideur ${ }^{1}$, B. Ortaç ${ }^{1,2}$, J.-B. Lecourt ${ }^{1}$, C. Chédot ${ }^{1}$, M. Brunel $^{1}$, \\ B. Chéron ${ }^{1}$, J. Limpert ${ }^{2}$, T. Tunnermann ${ }^{2,3}, \mathrm{Ph}$. Grelu ${ }^{4}$, M. Gicquel-Guézo ${ }^{5}$, \\ C. Labbé ${ }^{5}$, S. Loualiche ${ }^{5}$, Ph. Roussignol ${ }^{6}$, F. Sanchez ${ }^{7}$, H. Leblond ${ }^{7}$ \\ et A. Komarov 7,8 \\ ${ }^{1}$ Groupe d'Optique et d'Optronique, CORIA UMR 6614, Avenue de l'Université, \\ 76801 Saint Étienne du Rouvray, France \\ ${ }^{2}$ Friedlich Schiller University, Jena, Institute of Applied Physics, Jena, Germany \\ ${ }^{3}$ Fraunhofer Institute for Applied Optics and Precision Engineering, Albert Einstein-Str. 7, \\ 07745 Jena, Germany \\ ${ }^{4}$ Laboratoire de Physique de l'Université de Bourgogne, UMR 5027, 47870 Dijon, France \\ ${ }^{5}$ Laboratoire de Physique des Solides, INSA 20 avenue des Buttes de Coesme, \\ 35043 Rennes Cedex, France \\ ${ }^{6}$ Laboratoire Pierre Aigrain, École Normale Supérieure, Paris, France \\ ${ }^{7}$ Laboratoire POMA, UMR 6136, Université d'Angers, France \\ ${ }^{8}$ Institute of Automation and Electrometry, Russian Academy of Sciences, \\ Novosibirsk, Russia
}

\begin{abstract}
Résumé. Le premier laser à fibre dopé aux ions de terres rares fonctionna au tout début des années 60. Il fournissait quelques milliwatts autour de $1 \mu \mathrm{m}$. Les décades suivantes virent très peu d'améliorations tant du côté des laboratoires que du point de vue industriel. La dernière décennie (1995/2005) vit se concrétiser la seconde révolution des lasers à fibres. Déjà kilowatt en continu, ils atteignent désormais les $10^{13}$ watts $/ \mathrm{cm}^{2}$ avec des impulsions de la centaine de femtoseconde. Lors de cette présentation nous passerons en revue les potentialités des lasers à fibre. Nous décrirons les verrous technologiques qui ont été levés ces dix dernières années pour les régimes $\mathrm{CW}$ mais aussi femtosecondes. Nous montrerons également comment la prochaine génération de fibres optiques actuellement en développement permettra d'offrir des sources stables et de très haute puissance pour l'avenir proche.
\end{abstract}

\section{INTRODUCTION}

L'apparition de lasers fournissant des champs électromagnétiques intenses $\left[\mathrm{I}>10^{12} \mathrm{~W} / \mathrm{cm}^{2} ; \mathrm{E}>\right.$ $27,5 \mathrm{MV} / \mathrm{cm}$ ] a permis depuis maintenant 20 ans, l'avènement d'une nouvelle physique. En effet, l'ATI (Above Threshold Ionization) est l'un des tous premiers - et le seul ? - domaine de la théorie des champs ne pouvant pas s'interpréter par un développement perturbatif de la polarisation macroscopique induite. L'une des cousines de cette physique est la génération d'harmoniques d'ordres élevés (HOH-G). Elle permet depuis maintenant plus de 15 ans d'étendre la production de rayonnement cohérent vers les extrêmes limites UV du spectre électromagnétique [1]. L'ATI et l'HOH-G ont réellement pris leur essor au début des années 90 [2] lorsque les jalons théoriques y ont été clairement posés [3] et les sources lasers génératrices disponibles [4]. Par la suite s'en ait suivi tout un ensemble de méthodologies visant à comprendre et à améliorer le rendement des HOH-G [5]: optimisation de la focalisation du faisceau générateur IR et/ou de sa durée (direct or time gated sub-10fs pulses), influence des effets multi-atomes sur la propagation du rayonnement $\mathrm{HOH}$, influence et mise en forme de la phase du rayonnement $\mathrm{HOH}$ (Quasi Phase Matching, QPM), etc. Depuis le début des années 2000, les premières applications ont 
ainsi pû voir le jour: lithographie sub-20 nm, imagerie dans la fenêtre de l'eau (hv $\geq 300 \mathrm{eV})$ [6], analyse spectro-temporel des champs photo-électroniques [7].

D'autre part les champs optiques très intenses $\left[\mathrm{I}>10^{18} \mathrm{~W} / \mathrm{cm}^{2} ; \mathrm{E}>27,5 \mathrm{TV} / \mathrm{cm}\right]$ ont fourni des outils pour l'étude d'une physique relativiste 'presque' Table-Top [8]. L'accélération plasma relativiste par l'intermédiaire du champ de sillage produit par l'effet pondéromoteur de l'impulsion [9] n'en ait qu'une facette mais couplée à des accélérateurs de particules - que l'on ne peut pas qualifier de vraiment 'Table-Top' (!) - les premiers essais réussis de création de matière par la lumière laisse rêveur [10].

Parmi ces bouleversements scientifiques, nombres d'entre eux, si ce n'est tous, ont pour origine la possibilité de créer ces champs intenses; c'est à dire l'avènement du laser CPA ('Chirp Pulse Amplification') dont le leader est sans conteste actuellement la chaîne Ti-Sa amplifiée (et éventuellement accordable par OPA: Optical Parametric Amplifier). Pourtant, les problèmes technologiques de ce laser sont nombreux :

- non pompé directement par diode

- difficile à amorcer en régime impulsionnel stable

- réglages et alignements sensibles

- coût élevé

Loin de pouvoir régler chacun de ces inconvénients, on peut néanmoins s'interroger sur les possibilités du remplacement de ce laser pour certaines applications. Entre autres, pour celles nécessitant des champs lasers pas trop intenses (typ. I $<10^{14} \mathrm{~W} / \mathrm{cm}^{2}, \mathrm{E}<275 \mathrm{MV} / \mathrm{cm}$ ), les lasers à fibres semblent pouvoir être pris au sérieux. Un rapide calcul montre en effet que pour un faisceau de diamètre $50 \mu \mathrm{m}$ et une impulsion de $50 \mathrm{fs}$ de durée, l'énergie par impulsion satisfaisant cette limite, atteint $100 \mu \mathrm{J}$. A une cadence typique des lasers à fibres de $20 \mathrm{MHz}$, cela conduit à une puissance moyenne de $2 \mathrm{~kW}$; puissance qui tombe à $0,1 \mathrm{~W}$ à une cadence de $1 \mathrm{kHz}$. Bien qu'un tel laser à fibre n'existe pas encore, tout porte à croire qu'il devrait être disponible dans les années à venir.

Dans cet article, après avoir présenté un rapide état de l'art des performances des lasers à fibre en régime continu, nous discuterons avec plus de détails les succès récents des lasers à fibres à monoimpulsions brèves (c.à.d. délivrant des impulsions de quelques picosecondes à quelques dizaines de femtosecondes). Nous dresserons un panorama - non exhaustif - des meilleurs sources commercialisées que l'on trouve à l'heure actuelle sur le marché. Nous les classerons selon différents critères illustrant leurs performances énergétiques et temporelles. Les performances obtenues en laboratoire seront ensuite déclinées. Les différentes avancées et percées technologiques qui ont permis ces réussites seront rappelées. Hormis vis à vis de leurs caractéristiques énergétiques, les lasers à fibre ont connu également un réel progrès ces dix dernières années vis à vis des régimes plus ou moins exotiques qu'ils s'avèrent capable de délivrer avec une stabilité record et une aisance surprenante. En effet, nous montrerons que ces lasers offrent une souplesse inégalée pour la génération d'états liés, de régimes harmoniques ou encore d'impulsions paraboliques à dérive de fréquence linéaire. Enfin nous montrerons que ces lasers peuvent être parfaitement auto-démarrants sans aucun réglage de maintenance grâce à l'emploi d'absorbants saturables semiconducteurs; soit bien connus, car utilisant la technologie des multi-puits quantiques; soit plus novateurs, plus simples et semblant extrêmement prometteurs, à base de nanotubes de carbone. Les modélisations récentes menées par nos groupes et permettant d'interpréter les résultats obtenus et de prédire des optimisations futures seront également présentées. Basées sur des modèles vectoriels, ils permettent de suivre l'impulsion à tout instant et en tout point dans la cavité fibrée aussi bien depuis sa création que dans son régime établi. Nous statuerons sur les principaux verrous technologiques qui semblent pouvoir être levés avant la fin de la décennie afin de gagner un ou deux ordres de grandeur pour améliorer les caractéristiques (énergétiques et temporelles) de ces lasers à fibre à impulsions brèves. Enfin, une autre potentialité intéressante de ces lasers à fibre sera également rappelée. Elle concerne la possibilité d'optimiser la génération de rayonnement UV grâce à la génération d'harmonique d'ordre élevé par quasi-accord de phase dans un fibre à cœur creux modulé. 


\section{LES LASERS À FIBRE EN RÉGIME CONTINU (CW)}

Contrairement à tout autre type de laser solide pompé optiquement, les lasers à fibre utilisent la propagation guidée. Cet état de fait permet d'envisager des longueurs amplificatrices traversées par le faisceau laser inimaginables comparativement aux barreaux solides (même après multi-passages). En effet plusieurs centaines de mètres sont concevables sans aucune difficulté et des cavités laser de plusieurs kilomètres ont même été réalisées pour des besoins académiques d'études des régimes transitoires. La possibilité de doper le cœur de ces fibres par quasiment l'ensemble des ions de la troisième série de transition (famille des lanthanides ou aussi appelés terres rares) permet la génération d'une émission laser directe (sans conversion de fréquence) allant de moins de $400 \mathrm{~nm}$ ( $380 \mathrm{~nm}$ pour le $\mathrm{Pr}^{3+}$ ) à plus de $2.8 \mu \mathrm{m}$ ou $3 \mu \mathrm{m}$ (pour $\mathrm{l}^{\prime} \mathrm{Ho}^{3+} \mathrm{ou} \mathrm{l}^{\prime} \mathrm{Er}^{3+}$ respectivement). La disponibilités de diodes lasers de pompe pour quasiment l'ensemble de cette gamme, fait du laser à fibre à la fois le laser le plus compact et polyvalent au monde.

De part sa conception, le laser à fibre est: Non toxique (comparativement aux lasers $\mathrm{CO}_{2}$ et Excimer), à gestion thermique aisée (du fait de son rapport surface d'échange sur volume de gain élevé), monomode transverse $\left(\mathrm{M}^{2}\right.$ ne dépassant pas 1.5 pour les lasers à base de fibres optiques monomodes), à rendement élevé (puisque bien souvent à pompage directement par diode laser. On parlera aussi bien de rendement quantique que de rendement de conversion électrique/optique élevé), à faible logistique de maintenance ou service après-vente (puisque auto-aligné et scellé) et enfin à coût d'achat réduit (par conception technologique; bien que ceci reste encore à démontrer à puissance équivalente vis à vis d'autres types de laser).

Concernant les lasers à fibre monomode de puissance émettant en continu, les performances se sont améliorées de manière drastique ces quatre dernières années. Comme le montre la figure 1, après une progression constante mais de pente plus faible depuis le début des années 90 - progression impulsée pour l'essentiel par l'amélioration de la qualité des fibres monomode - la rupture de pente d'après le début des années 2000 est spectaculaire. Elle est due à une conjonction de plusieurs facteurs. Tout d'abord l'avènement de fibres double-gaine ('double-clad') où une première gaine extérieure entoure une seconde gaine interne avant le cœur dopé concentrique et monomode. Cette seconde gaine permet l'injection dans la fibre, de puissances de pompe conséquentes issues de lasers de puissance multimodes transverses. Ainsi des lasers de pompe de très haute puissance mais de faible brillance peuvent être

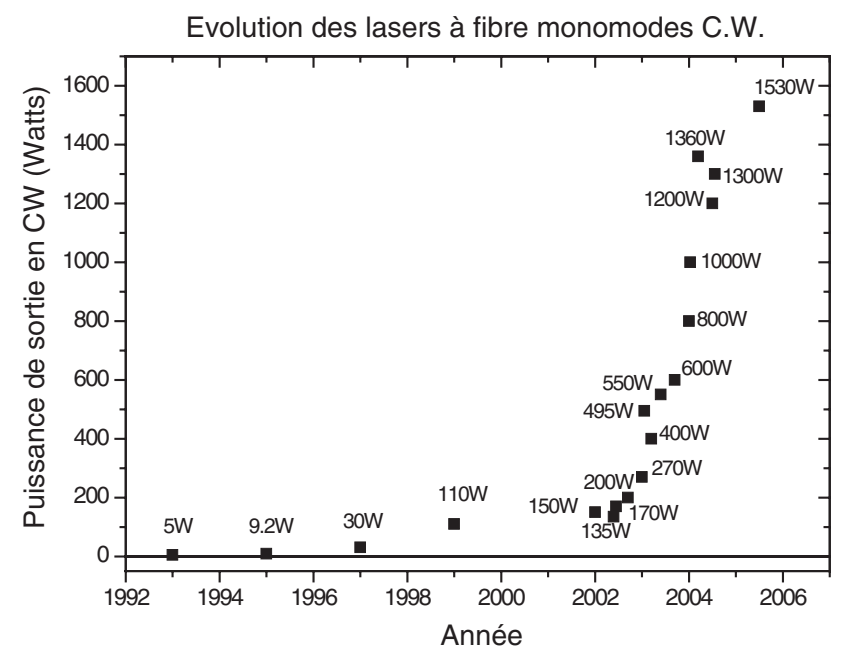

Figure 1. Evolution de la puissance de sortie émise en continu (C.W.) par les lasers à fibre depuis 15 ans. d'après [11]. 
couplées dans la fibre. Afin que ces énergies pompent le plus efficacement le cœur monomode, une brisure de la symétrie cylindrique de la seconde gaine doit être opérée. Ceci évite ainsi les rayons de pompe hélicoïdaux ne pompant jamais le cœur. Des fibres de forme en simple (ou double) D ont fait leur apparition. Des géométries rectangulaires, hexagonales ou encore en pétales ont ainsi révolutionné les limites de puissance couplée dans le cœur de ces fibres. Ensuite, l'avènement de diodes lasers de très hautes puissances (>100 watts) ont permis l'intégration de ces lasers à fibre de puissance en $\mathrm{CW}$. Enfin l'arrivée des fibres à fort diamètre de cœur (Large Mode Area fibers, ou LMA) pouvant restées monomodes par gestion du guidage constitua la $3^{\text {iéme }}$ révolution à la fin des années 90.

Ces fibres où le guidage monomodal est assuré par une gestion subtile de milieux de fort et bas indices peuvent être déclinées à 1 seule dimension (fibres dites de Bragg, [12]), ou à deux dimensions (fibres dites à cristal photonique ou PCF, [13]) ou encore grâce à une gaine d'air (fibres dites Air-Clad, [14]) ou un cœur d'air (fibres dites Air-Core ou Photonic Band Gap: PBG, [14]).

$\mathrm{Au}$ final, en régime continu, des puissances autour de $1500 \mathrm{~W}$ ont été générées et transportées par des laser à fibre, à l'heure d'aujourd'hui [11]. La limite de fusion de la silice étant estimée à plus de $4.5 \mathrm{~kW}$, rien n'interdit de penser que cette limite ne sera pas atteinte prochainement.

Ces avancées ont également des retombées pour les lasers à fibres à impulsions brèves. Permettant d'obtenir à la fois un management de la dispersion et une gestion des nonlinéarités en repoussant par exemple le seuil d'apparition des effets Brillouin, Raman et Kerr, ces fibres ont permis l'avènement de nouvelles sources cohérentes sub-picosecondes possédant des puissances crêtes extrêmement élevées, pouvant dépasser le MWatt.

\section{LES LASERS À FIBRE EN RÉGIME PULSES PICOSECONDES et SUB-PICOSECONDES}

\subsection{Régime mono-impulsionnel sub-picoseconde: vers l'impulsion à dérive de fréquence linéaire}

En régime mono-impulsionnel picoseconde et sub-picoseconde, le tableau suivant résume les percées technologiques de ces 15 dernières années c.à.d. depuis le renouveau des lasers à fibre. On notera la corrélation des dates avec celles de la figure 1. Modeste en 1993, les caractéristiques pulsées n'ont cessé de croître pour exploser depuis trois ans.

De nos jours, un peu moins d'une dizaine de firmes vendent de part le monde des produits lasers à fibre performants. La figure suivante en isole six en fonction de différentes performances bien que deux ou trois autres mériteraient sans conteste de figurer, comme par exemple MenloSystems (Allemagne), PolarOnyx (USA). On pourrait également citer Amplitude Technologies (France); cette dernière compagnie étant plus connue pour ces lasers solides Ti-Sa mais se lançant dans la technologie fibre avec succès depuis peu. Il est vrai que la croissance du marché des lasers à fibre est estimée à plus de $35 \%$ actuellement - contre une croissance de seulement $9 \%$ pour l'ensemble du secteur industriel laser. Ceci permet de prédire d'ici la fin de la décennie, un marché estimé à plus de $700 \times 10^{6} \$$ pour les lasers à fibre contre $3 \times 10^{9} \$$ pour l'ensemble des lasers [21].

Pour revenir à l'état de l'art de la recherche en laboratoire, citons que notre groupe a participé activement à ce renouveau des lasers à fibres à impulsions brèves depuis 2000. Après plusieurs réalisations sur les lasers à fibre continus et accordables, plus de $24 \mathrm{~nJ}$ avaient été extraits pendant 670 fs dès 2001 d'un oscillateur Ytterbium fibré utilisant la technologie double-clad [22]. A partir de ce même oscillateur non amplifié, des impulsions de $90 \mathrm{fs}$ et de plus de $5 \mathrm{~nJ}$ ont ensuite été générées en 2003 grâce à une gestion de la dispersion intra-cavité adéquate [16-a]. Avec une cadence de $18 \mathrm{MHz}$, la puissance moyenne dépasse les $100 \mathrm{~mW}$. Ensuite les choses se sont accélérées. Egalement en 2003 le groupe de Wise à Cornell aux USA annonce également $5 \mathrm{~nJ}$ mais pour des impulsions de seulement $50 \mathrm{fs}$ de durée puis $1.5 \mathrm{~nJ}$ sous $36 \mathrm{fs}$ [23]. Toutefois, dans ces 2 derniers lasers, l'allure spectrale est beaucoup plus chahutée. Fin 2004, Wise démontre la réalisation d'une source de 14 nJ par impulsion à partir du même oscillateur Yb [24]. Proche d'un régime parabolique précédemment démontré [25] 
Tableau 1. Etat de l'art fin 2004 des records sortant d'un oscillateur ou d'un amplificateur à fibre femtoseconde d'après [20].

\begin{tabular}{|c|c|c|c|}
\hline $\begin{array}{c}\text { Oscillateurs et Amplificateurs } \\
\text { à Fibres }\end{array}$ & $\mathbf{N d}$ & $\mathbf{E r}$ & $\mathbf{Y b}$ \\
\hline \multirow{4}{*}{$\begin{array}{c}\text { Résultats expérimentaux: } \\
\text { Record mondial de durée } \\
\text { (oscillateur): } \\
\text { Groupe, année } \\
\text { Puissance moyenne } \\
\text { correspondante : }\end{array}$} & & & \\
\hline & $\begin{array}{c}\mathbf{2 6 0 f s} \\
{[15-\mathrm{a}], 1993} \\
\mathbf{4} \mathbf{~ m W}\end{array}$ & $\begin{array}{c}\mathbf{6 5} \mathbf{f s} \\
{[15-\mathrm{b}], 2003} \\
\mathbf{1 1 0} \mathbf{~ m W}\end{array}$ & $\begin{array}{c}\mathbf{9 0 \mathbf { f s }} \\
{[16-\mathrm{a}], 2003} \\
\mathbf{8 5} \mathbf{~ m W}\end{array}$ \\
\hline & $\begin{array}{c}\mathbf{4 2} \mathbf{f s} \\
{[16-\mathrm{b}], 1993} \\
\mathbf{7 2} \mathbf{~ m W}\end{array}$ & $\begin{array}{c}\mathbf{6 3} \mathbf{f s} \\
{[17-\mathrm{a}], 1995} \\
\mathbf{2} \mathbf{~ m W}\end{array}$ & $\begin{array}{c}\mathbf{5 2} \mathbf{f s} \\
{[17-\mathrm{b}], 2003} \\
\mathbf{6 5} \mathbf{~ m W}\end{array}$ \\
\hline & $\begin{array}{c}\mathbf{3 0 0} \text { fs }(\text { tunable: } 1054-1128) \\
{[18-\mathrm{a}], 1995} \\
\mathbf{8} \mathbf{~ m W}\end{array}$ & & \\
\hline \multirow{3}{*}{$\begin{array}{l}\text { Record mondial de durée } \\
\quad \text { (amplificateur) : } \\
\text { (Energie, cadence, puiss. } \\
\left.\text { moyenne, } P_{\text {pompe }}\right)\end{array}$} & & & $\begin{array}{c}120 \mathrm{fs} \\
{[18-\mathrm{b}], 2003} \\
6 \mathrm{~nJ}, 50 \mathrm{MHz}, \mathbf{3 0 0} \mathbf{~ m W}, 1 \mathrm{~W}\end{array}$ \\
\hline & & & $\begin{array}{c}\mathbf{2 5 0} \mathbf{f s} \\
{[19-\mathrm{a}], 2003} \\
400 \mathrm{~nJ}, 75 \mathrm{MHz}, \mathbf{7 6} \mathbf{W}, 180 \mathrm{~W}\end{array}$ \\
\hline & & & $\begin{array}{c}\mathbf{2 2 0} \mathbf{f s} \\
{[19-\mathrm{b}], 2001} \\
220 \mathrm{~nJ}, 1 \mathrm{MHz}, \mathbf{5 , 5} \mathbf{W}, 18 \mathrm{~W}\end{array}$ \\
\hline
\end{tabular}

(cf. plus loin), ces impulsions présentent un spectre beaucoup plus propre. Les impulsions compressées descendent à 85 fs. Enfin ce groupe annonce à CLEO/QELS en 2005, une version étirée puis préamplifiée par un double étage de fibres $\mathrm{Yb}$, et enfin amplifié par un troisième tronçon de fibre $\mathrm{Yb}$. Un acousto-optique permettant de descendre la cadence entre $3 \mathrm{MHz}$ et $188 \mathrm{kHz}$ et d'isoler l'amplificateur final de l'émission spontanée amplifiée est également utilisé. Au final, des impulsions de $800 \mathrm{~nJ}$, $150 \mathrm{fs}$ autour de $1030 \mathrm{~nm}$ sont délivrées à une cadence de $188 \mathrm{kHz}$ [26-a]. Pratiquement en parallèle, Martin Fermann et son groupe d'IMRA America (cf. Fig. 2) annonce une version quasi-similaire de système CPA fibré délivrant $100 \mu \mathrm{J}$ par impulsion de $650 \mathrm{fs}$ de durée après recompression [26-b]. L'étage de recompression n'est pas fibré mais constitué de réseaux massifs. La nouveauté réside ici en deux points majeurs: d'une part l'emploi d'une fibre à cristaux photoniques (PCF) dopée Yb pour constituer l'étage d'amplification de puissance et d'autre part une gestion radicalement novatrice de la dispersion entre les étages d'étirement et de compression. En effet, un fort désaccord voulu entre la dispersion d'ordre 3 de l'étireur et du compresseur est compensé directement par l'automodulation de phase de la fibre amplificatrice PCF. Cette technique engendre des impulsions "cubicons" à profil d'autocorrélation exponentielle décroissante et à spectre asymétrique. Leur nom illustre la gestion de la dispersion d'ordre 3 et s'inspire des impulsions auto-similaires (ou similaritons) aux profils spectral, temporel et de phase quadratiques - c.à.d. parabolique (cf plus loin).

Dans le domaine pulsé femtoseconde de puissance les plafonds ont été dépassés également par le groupe de Andreäs Tunnermann et de Jens Limpert respectivement à la Friedrich-Schiller University et au Fraunhofer Institute à Jena. Pour ne citer que les réalisations les plus marquantes et les plus récentes - on pourra aussi se référer à l'article de revue publié en 2005 dans J. Phys. B: At. Mol. Opt. Phys concernant la renaissance et les perspectives futures des lasers à fibres, [11] - nous évoquerons 3 résultats. $131 \mathrm{~W}$ de puissance moyenne ont été délivrées par des impulsions de $1,8 \mu \mathrm{J}$ à partir de fibres à cristaux photoniques large mode (LMA fiber) [27]. Ces fibres ont été utilisées dans une configuration CPA à 2 étages d'amplification, la recompression se faisant à travers des réseaux massifs utilisés en 

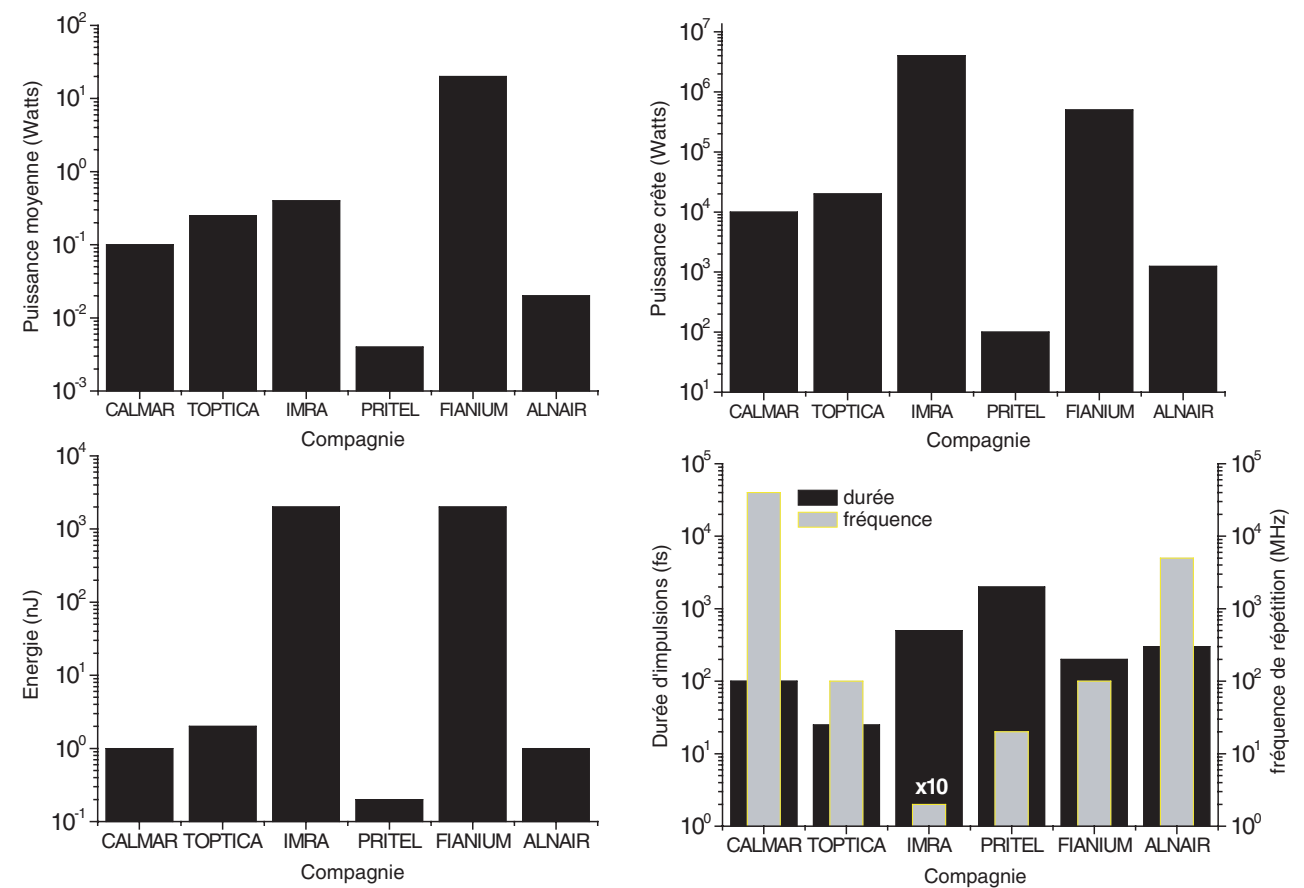

Figure 2. Caractéristiques des lasers à fibres à impulsions ultra-brèves fournies par les principales compagnies mondiales. Les différentes caractéristiques ne sont pas corrélées entre elles mais relèvent environ à chaque fois des meilleurs lasers fournies par la compagnie.

transmission spécifiquement conçus pour cette application. Ces impulsions de $220 \mathrm{fs}$ ont ensuite été descendues à $60 \mathrm{fs}$ (tout en gardant une énergie par impulsion de $1 \mu \mathrm{J}$ ) grâce à un étage supplémentaire d'étireur-compresseur utilisant respectivement un faible tronçon $(4 \mathrm{~cm})$ de fibre LMA et un système multipass à base de miroirs chirpés [27]. L'inconvénient de ces sources est que l'impulsion initiale (seed) est générée à partir d'un oscillateur faible énergie et faible bruit à base de cristaux solides. La chaîne laser ainsi constituée n'est donc pas entièrement fibrée. Un pas supplémentaire vers les systèmes totalement intégrés en fibres optiques a alors été fait lorsque ce même groupe, avec qui nous collaborons étroitement pour le développement d'oscillateurs fibrés [28], a récemment [29] réalisé une chaîne (oscillateur + amplificateur) totalement fibrée et auto-démarrante. Seuls les compresseurs du CPA restent actuellement massifs pour des raisons de tenue au flux. Cette chaîne a permis de sortir plus du microjoule sur des impulsions de $240 \mathrm{fs}$ à une cadence de $17 \mathrm{MHz}$. On peut résumer les résultats des travaux de Jena par les trois séries de caractéristiques suivantes : - $60 \mathrm{fs}, 1.1 \mu \mathrm{J},<45 \mathrm{~W}>$, système entièrement fibré

- $220 \mathrm{fs}, 1.79 \mu \mathrm{J},<131 \mathrm{~W}>$, système contenant des ensembles non fibrés

- $240 \mathrm{fs}, 1.2 \mu \mathrm{J},<21 \mathrm{~W}>$ système fibré à maintien de polarisation

Ces trois configurations de sources contiennent des fibres à cristaux photoniques (PCF) et peuvent également contenir un absorbant saturable afin d'initier le régime impulsionnel mode-locking du laser. La figure 3 donne le schéma de principe d'un tel système atteignant et délivrant le micro-Joule fibré dans le cas d'un système à maintien de polarisation et utilisant un absorbant saturable pour le démarrage.

Il faut noter que les différentes avancées et percées technologiques qui ont permis ces réussites tournent autour des fibres double-gaine, des fibres à cristaux photoniques mais dépendent également des techniques novatrices qui ont débouchées ces dernières années sur le développement de diodes lasers semiconductrices de puissance performantes. En effet ces lasers à fibre utilisent des diodes de 


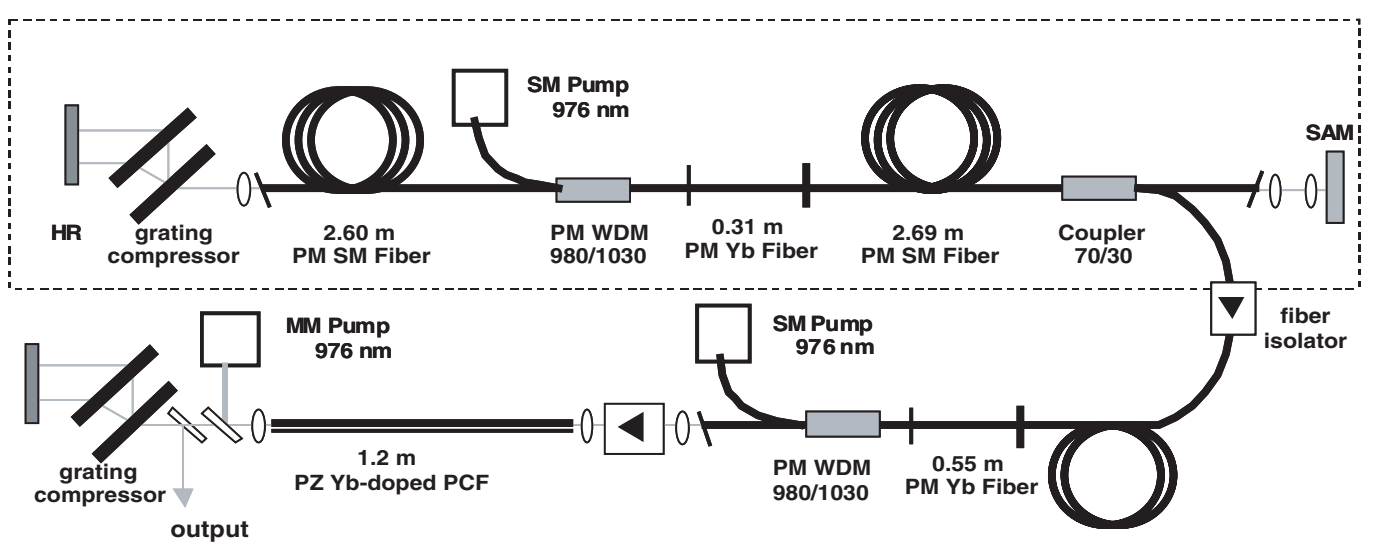

Figure 3. Schéma de principe d'un laser oscillateur fibré à maintien de polarisation et auto-démarrant par un absorbant saturable miroir de fond de cavité (SAM) tel que développé par Jena [29] et présenté à Photonics West 2006. Les impulsions sont étirées, pré-amplifiées puis amplifiées également par fibre. Seul le compresseur reste en air libre.

pompes de puissances à $976 \mathrm{~nm}$ ou $980 \mathrm{~nm}$ capables de délivrer couramment des puissances supérieures à 30 voire 50 watts en bout de fibres multimodes.

\subsection{Régimes multi-impulsionnels sub-picosecondes \& paraboliques}

L'intérêt des lasers à fibre délivrant des faisceaux en limite de diffraction ne s'arrêtent pas à leur compacité (donc faible poids, faible encombrement) et à leur grande stabilité (auto-alignement, reproductibilité) ni à leur puissance. Ces atouts en font pourtant d'ores et déjà des chaînes lasers à faible coût très intéressantes pour certaines applications scientifiques de spectroscopie linéaire, non linéaire, microscopie CARS ou FLIM et/ou d'imagerie confocale où la brillance du faisceau est primordial. En effet, ces sources ont également montré des comportements stables et tout à fait étonnants en régimes d'impulsions liées (bound states) [30] ou harmoniques [31].

Dans le premier cas, le laser génère des paires ou triplets d'impulsions liées c.à.d. dont la séparation temporelle (pouvant aller de quelques durées d'impulsions à plus d'un millier de fois la durée de chaque impulsion) est rigoureusement stable pendant des heures. Ceci se traduit essentiellement par un spectre optique modulé du fait d'une phase fixe entre impulsions occupant des valeurs quantifiées, $0, \pi / 2, \pi$ ou autres en fonction de la dispersion induite par la cavité [32]. Un tel résultat de deux impulsions liées est présenté à la Fig. 4. La route vers l'établissement de ce régime peut s'avérer complexe tant du point de vue expérimentale que du point de vue des explications théoriques. Dans le premier cas, nous entendons par la que plusieurs paramètres peuvent permettre d'influencer l'obtention d'un tel régime. La puissance de pompe et les caractéristiques de l'absorbant saturable, qu'il soit réel (généralement à base de multi-puits quantiques, cf. plus loin) ou virtuel (c.à.d. utilisant le principe de la rotation non-linéaire de la polarisation [33]), sont les principales variables d'influence. Notons en revanche que ces régimes peuvent apparaître aussi bien en régime solitonique de dispersion moyenne de cavité anormale qu'en régime d'impulsion étirée à dispersion moyenne de cavité normale. Un train commun expérimental à ces régimes est leur très grande stabilité. L'absence de pré ou post-impulsion est également un point positif. Des niveaux de bruits records extrêmement faibles $(<0.1 \%)$ en terme de fluctuations d'amplitudes ont pu être enregistrés lorsque ces lasers basculent en régime d'impulsions liées. Ces faibles bruits sont largement comparables si ce n'est supérieurs à ceux obtenus avec certains lasers solides pompés par diodes. Ces niveaux de bruit peuvent même être inférieurs à celui du système de pompage utilisé. En dehors de l'isolation par la fibre, on peut penser que le laser optimise simplement ce régime pour 

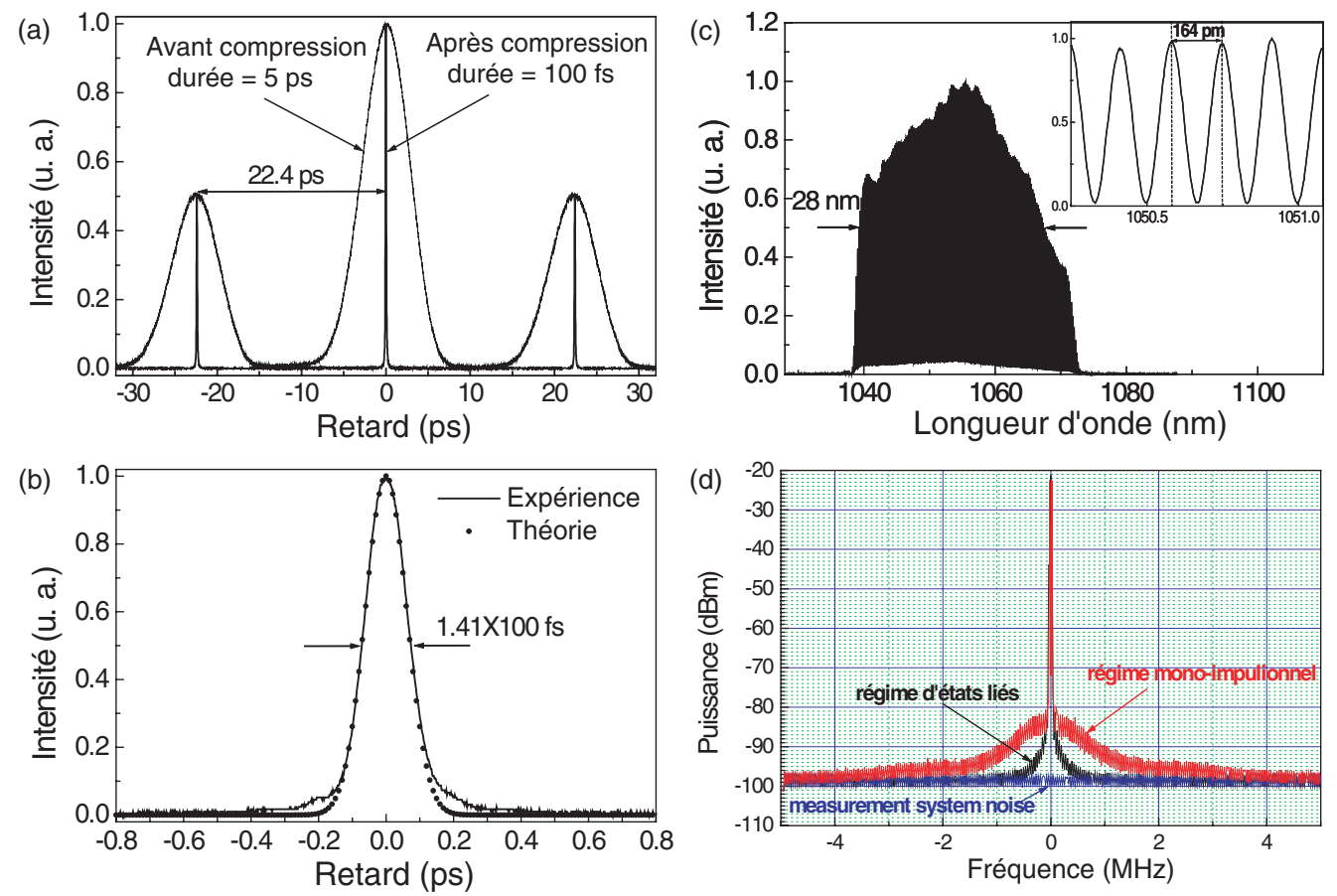

Figure 4. Caractéristiques d'un régime de deux impulsions liées en phase délivrées par laser à fibre monomode double gaine dopé Yb. (a) Trace d'autocorrélation du doublet d'impulsions liées. Les traces fines sont les impulsions compressées. Un zoom d'une impulsion compressée est présentée en (b). Le spectre optique modulé caractéristique de ce type de régime est en (c). La modulation sur quasiment $100 \%$ dénote la parfaite stabilité de ce régime. Cette stabilité est également quantitativement qualifiée par le spectre basse fréquence en (d).

minimiser ses dissipations et ses interactions avec l'extérieur. Des modélisations plus poussées ont été entreprises en prenant en compte uniquement les nonlinéarités de Kerr de la fibre optique (en plus des phénomènes de gain saturé et de dispersion) et permettent de retrouver exactement ce genre de régime (cf. plus loin).

Dans le second cas (harmonique), l'impulsion se scinde en un groupe équi-réparti temporellement. Dans le spectre radio-fréquence, on observe l'apparition d'une nouvelle fréquence fondamentale pour le laser, multiple entier de la fréquence initiale. Nous avons démontré en 2005 des régimes harmoniques pouvant dépasser $2 \mathrm{GHz}$ avec un laser à fibre fonctionnant à $1 \mu \mathrm{m}$ [31]. Il faut noter néanmoins que ces régimes multi-impulsions (liées ou harmoniques) se répartissent l'énergie disponible abaissant par làmême l'énergie par impulsion. Toutefois ces régimes peuvent rester stables à fort pompage moyennant un management de leur dispersion adéquate.

Un autre régime extrêmement intéressant a également été mis en évidence. Il s'agit du régime d'impulsions à profil spectral, profil temporel et profil de phase parabolique. L'intérêt de ce régime réside en cette dernière propriété. En effet, faisant parti des phénomènes physiques classables dans la famille des objets auto-similaires (ou similaritons), la propagation d'une telle impulsion conserve une distribution quadratique sur chacun des trois profils. Le point le plus notable de ce phénomène est qu'il se conserve également sous amplification de l'impulsion. La phase quadratique imposant un paramètre de dérive de fréquence (chirp) linéaire, une telle impulsion amplifiée peut être parfaitement recompressée théoriquement en limite de Fourier après amplification. Nous avons récemment démontré la réalisation d'un laser à fibre fournissant de telles impulsions paraboliques d'énergies $3.2 \mathrm{~nJ}$ et de durées 6,4 ps [34-a]. Le spectre est parfaitement parabolique alors que la trace d'autocorrélation a 
une allure plutôt gaussienne. Une recompression extra-cavité a permis d'obtenir des durées de 140 fs. Un léger piédestal sur l'impulsion recompressée prouve que le régime généré n'est pas optimisé en similariton. Ce dernier régime étant un régime asymptotique, nous avons récemment montré par calcul numérique, qu'un régime parfaitement auto-similaire ne semble pas pouvoir être obtenu en sortie d'un oscillateur, qui est par définition périodique.

Une autre curiosité obtenue récemment au laboratoire est la génération d'un régime de deux puis trois impulsions liées et à profils paraboliques [34-b]. Le régime auto-similaire étant normalement adéquate pour supporter de fortes amplifications, il est intéressant de voir que le laser choisit toutefois le régime d'impulsions liées - tout en gardant un profil parabolique - lorsque à la foi le pompage et les pertes de sortie du laser augmentent. Ces dernières sont évaluées à plus de $75 \%$ dans notre cas. Des modélisations numériques sont actuellement en cours pour tenter d'expliquer la hiérarchie des régimes obtenus en fonction des paramètres du laser.

\subsection{Auto-démarrage des lasers à fibre à impulsions brèves}

L'inconvénient des lasers précédents est qu'ils utilisent très souvent le phénomène de rotation nonlinéaire de la polarisation (RNLP) comme absorbant saturable virtuel (ASV) pour former le régime impulsionnel de verrouillage de mode. Ceci rend le laser pas nécessairement auto-démarrant lorsque le pompage augmente. Un réglage subtil suivant une procédure précise est alors nécessaire pour démarrer le laser en régime impulsionnel. Si ce point n'est pas trop problématique pour un laser utilisé par des scientifiques rompus aux techniques de l'optronique et de la photonique, il peut devenir un réel inconvénient pour un utilisateur peu formé aux lasers. Si le monde des laboratoires scientifiques pourrait encore se payer le luxe d'un technicien spécifique pour de telles sources fibrées à impulsions ultrabrèves, ceci devient inacceptable pour un industriel. La nécessité d'amorcer au moins le démarrage et éventuellement de stabiliser et sculpter l'impulsion ultra-courte par un absorbant saturable réel est donc une priorité. C'est dans ce but que nous avons entrepris également dans notre groupe des études de faisabilité et de qualification d'absorbants saturables réels (ASR) capables de former et de supporter des impulsions brèves. Différents types d'absorbants saturables ont été testés au laboratoire. Ils appartiennent tous à la famille des semiconducteurs. En effet les absorbants saturables organiques (types $\mathrm{BDeN}$ ) ou à base de cristaux solides (types $\mathrm{Cr}^{4+}$ ) présentent des temps de relaxation trop longs ( $>10 \mathrm{~ns}$ ) pour pouvoir espérer initier et maintenir un régime d'impulsions pico ou sub-picosecondes. Un semiconducteur typique ayant également des temps de relaxation de l'ordre de la nanoseconde, le même problème se pose. Nous avons donc préférentiellement utilisé des semiconducteurs à faible temps de relaxation ( $\leq 500 \mathrm{fs}$ ). Ceux-ci furent obtenus de deux manières différentes. Tout d'abord des multipuits quantiques InGaAs/InP furent utilisés [35-a]. Ces multipuits quantiques étaient dopés en ions Fer in-situ c.à.d lors de la croissance par épitaxie. L'ion Fer étant incorporé en centre profond dans la matrice, il accélère le temps de recombinaison des excitons. Des structures en transmission (SESAT: semiconductor S.A. used in Transmission) et en micro-cavité résonnante ou en désaccord de résonance de type Fabry-Pérot (SESAM: semiconductor S.A. mirror) ont été utilisées. Seules les structures en micro-cavités ont donné des régimes de verrouillage de mode honorables [35-b]. Des impulsions allant de moins de $300 \mathrm{fs}$ à plus de 2.5 ps ont pu être générées en fonction du régime de dispersion de la cavité. Les énergies et puissances moyennes obtenues jusqu'alors sont relativement médiocres puisque respectivement de l'ordre de la centaine de picojoules et du milliwatt. La disponibilité de structures A.S. absorbantes autour de $1.5 \mu \mathrm{m}$ nous ont orientés vers des expérimentations utilisant un laser à fibre dopé erbium émettant dans la bande des télécommunications fibrées [1530 nm - $1560 \mathrm{~nm}$ ]. $\mathrm{Ne}$ disposant pas de sources fibrées doubles gaines à ces longueurs d'ondes nous avons opté pour l'emploi de diodes de pompes monomodes transverses - donc de puissance limitée - pour l'étude de ces démonstrateurs. Ceci explique pour une très grande part les niveaux d'énergies obtenus assez faibles à ce jour. L'emploi de phénomènes de polarisation dans la cavité de l'oscillateur, soit en inclinant le SESAT à l'angle de Brewster soit en incorporant un polariseur avant le SESAM, nous a permis 
de diminuer considérablement le bruit d'amplitude et d'une manière générale la qualité des impulsions par la suppression d'un quelconque piédestal [35-b].

Une toute autre famille d'absorbants saturables semiconducteurs a également été testée sur l'oscillateur à base de fibre dopée $\mathrm{Er}^{3+}$. Il s'agit de nanotubes de carbone, NTC [35-c]. Cette phase cristalline semiconductrice du carbone a révélé récemment ces propriétés en optique non-linéaire depuis que le groupe de Set et de Yamashita ont démontré et breveté la fonction A.S. et réalisé les tous premiers lasers à fibre à verrouillage de phase incorporant un A.S. à base de NTC (ou SAINT: Saturable Absorber Incorporated NanoTubes) [36]. Notons que ce groupe a d'ailleurs constitué une compagnie pour vendre ces lasers à fibre: le groupe ALNAIR Corp. (cf. Fig. 2). Les intérêts majeurs de ce genre d'A.S. sont au moins au nombre de deux. Tout d'abord il est de nos jours extrêmement simple de fabriquer des NTC. Disposés en solution, il est alors encore plus simple de constituer un SAINT puisque quelques gouttes déposés sur un miroir Au ou Ag suffisent. De plus il faut savoir que les NTC ont une limite de tenue au flux non encore clairement connue lorsqu'ils se trouvent en suspension dans une solution et plus de 100 fois plus élevée que celle des multi-puits quantiques lorsqu'ils sont déposés sur un substrat. Des fluences records de seuil de dommage optique de l'ordre de $32 \mathrm{~mJ} / \mathrm{cm}^{2}$ ont en effet été mesurées pour de telles structures. Il existe toutefois un inconvénient majeur (inhérent à leur composition) à l'emploi de ces SAINTs. Il s'agit de leur inhomogénéité de surface. Les NTC étant des cylindres creux de carbone de 1 à $1.5 \mathrm{~nm}$ de diamètre - ce qui fixe le 'band gap' - et allant de $100 \mathrm{~nm}$ à plus de 10 à $100 \mu \mathrm{m}$ de longueur, ces véritables 'plats de spaghettis' déposés sur un miroir, n'offrent pas vraiment ce que l'ont peut appeler une surface homogène. Ceci est d'autant plus vrai que ces spaghettis se forment en tas plus ou moins compacts, formant des sortes de dépôts comme des traces de suies sur le miroir ainsi revêtu. Des techniques de 'spin-coating' n'améliorent pas sensiblement l'homogénéité de surface pour le moment. En revanche, lorsque le faisceau laser est proprement aligné sur un agglomérat plus ou moins homogène de tels SAINTs, il s'avère que le laser est remarquablement bien verrouillé en phase. Des impulsions allant de 880 fs à 1.9 ps ont ainsi pu être obtenus par notre groupe en fonction du régime de dispersion moyenne de la cavité [35-c]. Des puissances moyennes de l'ordre de $3.5 \mathrm{~mW}$ pour des énergies par impulsion un peu inférieur à 200 pJ ont été obtenues. Le démonstrateur étant le même que pour les puits quantiques, la limitation est toujours celle de pompe. Des expérimentations sont actuellement en cours avec une diode de pompe monomode transverse délivrant une puissance de 1/2 watt. D'autres campagnes d'expérimentations sur un laser à fibre double-clad co-dopée Er-Yb avec une puissance de pompe de 8 watts doivent également avoir lieu avant la fin de l'année dans le groupe de François Sanchez / Laboratoire POMA à Angers. L'un des principaux avantages apparemment de l'emploi de ces structures est l'obtention d'un laser parfaitement stable avec une fluctuation d'amplitude extrêmement basse $(<0.5 \%)$ sans atteindre pour autant les record obtenu en RNLP à $1040 \mathrm{~nm}$ (cf plus haut). Ceci semble due pour une grande part à la surface parfaitement réfléchissante composée d'une monocouche $\mathrm{Au}$ ou $\mathrm{Ag}(\mathrm{R}>99.5 \%)$ du SAINT. La figure 5 présente une trace temporelle (à gauche) d'auto-démarrage du laser en régime de verrouillage de modes obtenue avec un SESAT, SESAM ou SAINT. Dans tous les cas, le laser démarre vers un transitoire d'un régime déclenché (type Q-switch) avant le régime de 'mode-locking'. La transition entre ces deux régimes semble obligatoirement passer par une impulsion géante particulièrement notable sur la figure. Ni son origine ni les paramètres influençant cette dernière ne sont proprement cernés ni compris à l'heure d'aujourd'hui.

\subsection{Modélisations des régimes sub-picosecondes}

Nous avons mené différentes modélisations de nos lasers à fibre verrouillés en phase. Pour l'instant, la plupart ont eu pour but d'expliquer l'ensemble des régimes obtenus avec le laser à fibre doubleclad dopé $\mathrm{Yb}$ : le régime mono-impulsionnel, les régimes d'impulsions liées, les régimes d'impulsions harmoniques et enfin les régimes d'impulsions paraboliques. Tous ces régimes sont basés sur l'emploi d'un Absorbant Saturable Virtuel (ASV): la Rotation Non-Linéaire de la Polarisation [33]. Nos modèles sont donc parfaitement résolus en polarisation et s'appuient sur les Equations de Schrödinger Non 

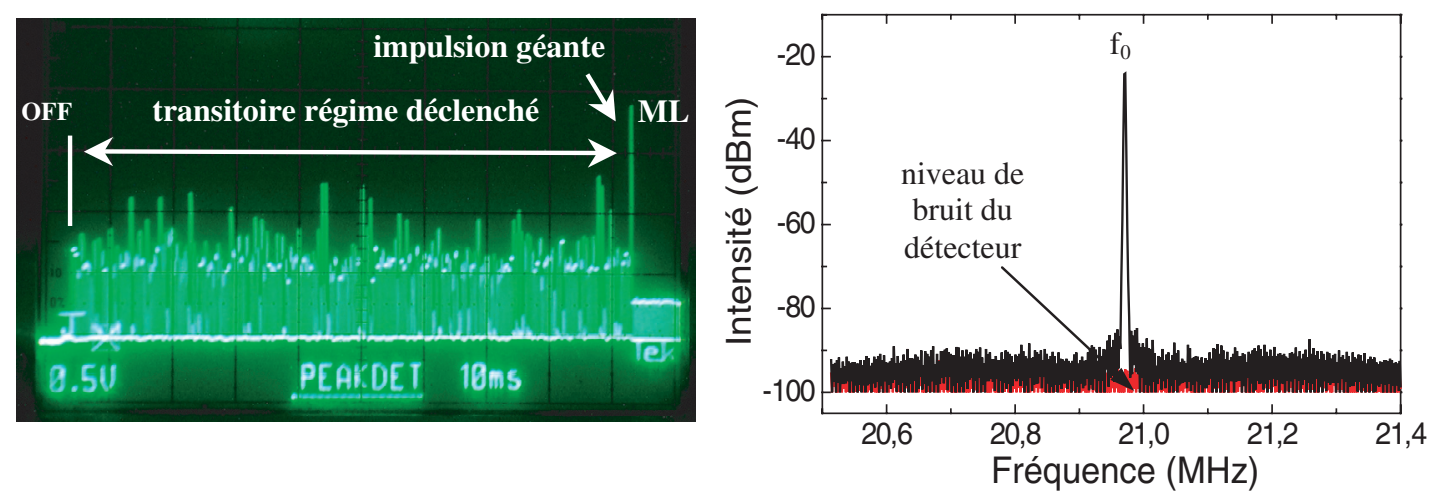

Figure 5. Transitoire de démarrage vers un régime de verrouillage de mode (ML) auto-démarrant dans le cas de l'emploi d'un A.S. de type SESAT, SESAM ou SAINT (gauche). Spectre radio-fréquence autour du fondamental $\left(\mathrm{f}_{0}\right)$ du laser à fibre dopée $\mathrm{Er}^{3+}$ en régime de verrouillage de mode auto-démarrant par A.S à base de nanotubes de carbones : SAINT (droite).

Linéaires (ESNL) et Equations de Ginzburg-Landau (EGL). Ils permettent de prendre en compte les différentes nonlinéarités de la fibre optique: automodulation de phase (SPM, $\Gamma$ ) et modulation de phase croisée (XPM, 2/3 1/3 $\Gamma$ ), gain saturé $(\mathrm{g})$ du milieu laser, filtrage spectral passif $\left(\beta_{\mathrm{p}}^{\prime}\right)$ des éléments et actif $\left(\beta{ }_{\mathrm{a}}\right)$ imposé par la bande de fluorescence du dopant. Ils prennent en compte les différentes dispersions de chaque tronçon de la cavité (fibres et réseaux de diffraction-compensation). Les dispersions à l'ordre $2\left(\beta_{2}\right)$ et $3\left(\beta_{3}\right)$ sont généralement prises en compte. La propagation de nos équations permettent de modéliser rigoureusement l'évolution de la polarisation dans la cavité. Le passage des différentes lames de phase quart-d'onde, demi-onde et du polariseur est traité par le formalisme des matrices de Jones. La valeur de la biréfringence linéaire $(\gamma)$ de chaque fibre optique est issue de la mesure de la longueur de battement des fibres [37]. Enfin les différentes pertes aux interfaces et/ou dioptres air-fibres ainsi que provenant des soudures entre fibres sont également incluses dans la modélisation. Les équations ont les formes suivantes :

$$
\begin{aligned}
& i \partial_{z} u+\gamma u+\frac{\beta_{2}}{2} \partial_{t}^{2} u+\Gamma\left(u|u|^{2}+\frac{2}{3} u|\mathrm{v}|^{2}+\frac{1}{3} \mathrm{v}^{2} u^{*}\right)=i\left(g+\beta^{\prime} \partial_{t}^{2}\right) u \\
& i \partial_{z} \mathrm{v}-\gamma \mathrm{v}+\frac{\beta_{2}}{2} \partial_{t}^{2} \mathrm{v}+\Gamma\left(\mathrm{v}|\mathrm{v}|^{2}+\frac{2}{3} \mathrm{v}|u|^{2}+\frac{1}{3} u^{2} \mathrm{v}^{*}\right)=i\left(g+\beta^{\prime} \partial_{t}^{2}\right) \mathrm{v}
\end{aligned}
$$

Les enveloppes $\mathrm{u}$ et $\mathrm{v}$ des champs optiques constituent les composantes du champ réel se propageant après décomposition sur une base orthogonale de deux polarisations orthogonales rectilignes. Ces modèles permettent de suivre l'impulsion à tout instant et en tout point dans la cavité fibrée aussi bien depuis sa création à partir de bruit ou d'impulsion_ansatz que dans son régime établi. Intégrées par la méthode du 'pas séparé' (Split-Step method), la simulation de plusieurs dizaines de milliers de tours de cavité peut s'avérer nécessaire pour préciser la convergence du régime entretenu. Les domaines temporel et spectral comportent un minimum de 2048 points. Outre les états de polarisation exacts le long d'une impulsion (temporellement ou spectralement), nos modèles permettent de préciser les cartographies des régimes obtenus en fonction de l'orientation des lames de phase 1/4 et 1/2 ondes. L'ensemble de ces cartographies reproduit avec beaucoup de similitude les régimes observés expérimentalement. La fig. 6 illustre la formation numérique d'un 'bound-state' de deux impulsions liées. 


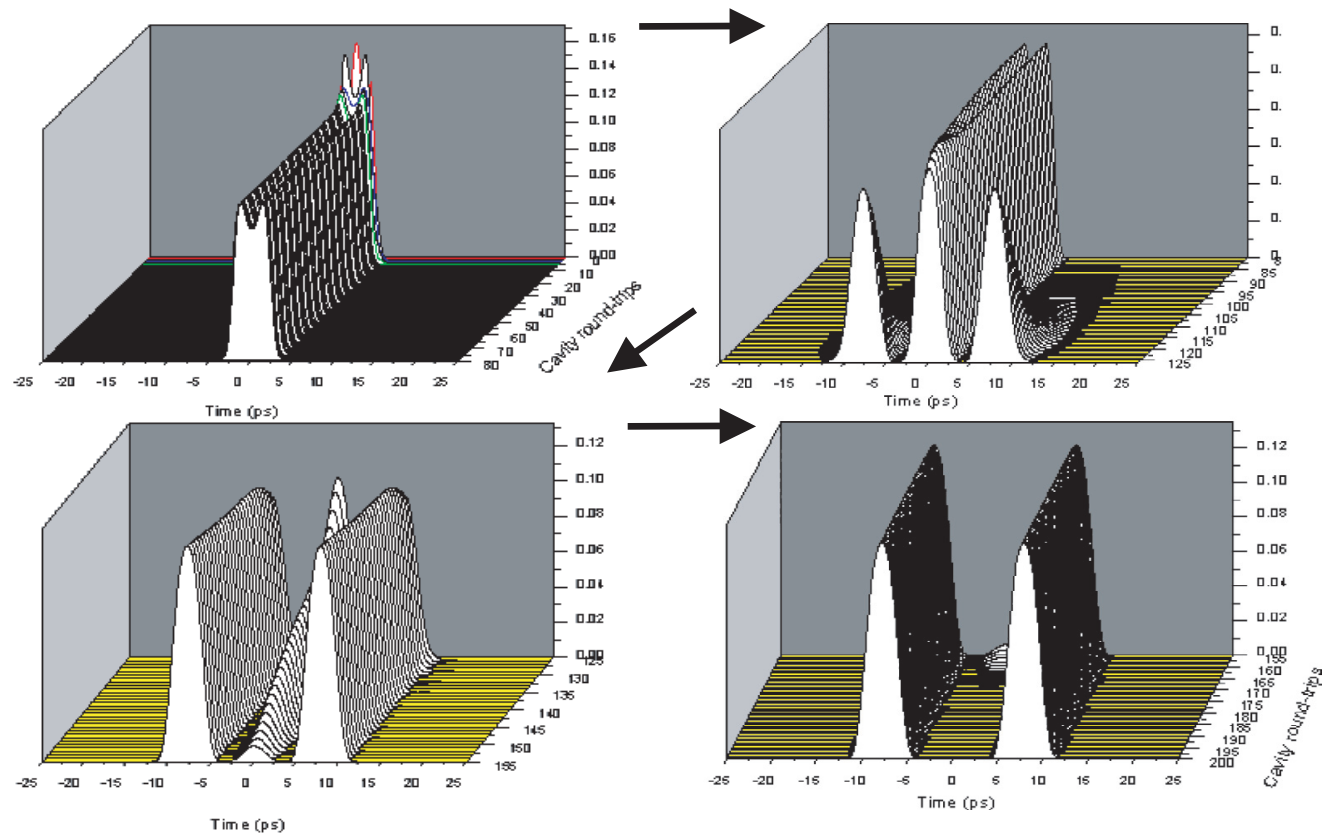

Figure 6. Simulation numérique de la formation d'un 'bound-states' de deux impulsions liées á partir d'une $\operatorname{sech}^{2}(t / \tau)$ quelconque. Au bout de 200 tours, la paire est entièrement formée et stable.

\section{CONCLUSIONS \& PERSPECTIVES}

Remarquons que ces oscillateurs entièrement fibrés peuvent trouver un intérêt immédiat dans l'optimisation de la génération d'harmoniques élevés (HOH-G) dans le cadre des études menées par le groupe de Kapteyn et Murnane [38]. L'amélioration du rendement HOH-G est réalisée via l'injection du faisceau laser dans une fibre à cœur creux emplie de gaz. La modulation spatiale du cœur joue le rôle de QPM entre les harmoniques générées et le signal pompe. Or l'emploi de ces fibres est actuellement sérieusement envisagé pour recompresser les impulsions en fin de chaîne laser fibré. Le soudage d'une telle fibre est alors très simple, avec l'avantage principal d'un rendement de couplage optimal quasi sans perte puisque fibre à fibre. La chaîne laser à fibre générant directement en bout de fibre des harmoniques élevées est-elle pour demain?

\section{Références}

[1] On exclut ici les sources X générées par LEL dont les techniques de génération sortent du cadre technique que se sont fixé ici les auteurs

[2] Bien que des études sur des domaines connexes aient été menées par des précurseurs bien avant; cf. Agostini et. al. IEEE JQE-4, 677 (1968); PRL 42, 1127 (1979); Lompré et. al. JOSA B 2, 1906 (1985). Pour revue, cf. Protopapas et.al. Rep. Prog. Phys 60, 389 (1997); Burnett et. al. J. Phys. B: At. Mol. Opt. 26, 561 (1993)

[3] P.B. Corkum, PRL 71, 1994 (1993); M. Ivanov et. al. PRL 74, 2933 (1995); M. Lewenstein et. al. Phys. Rev. A 49, 2117 (1994); M. Lewenstein et. al. Phys. Rev. A 52, 4747 (1995)

[4] Pour une revue, cf. par ex. Brabec et. al. Rev. Mod. Phys. 72,545 (2000); P. Agostini et. al. Rep. Prog. Phys 67, 813 (2004) 
[5] P. Balcou et. al. J. Phys. B: At. Mol. Opt. Phys. 25, 4467 (1992); Ph. Antoine et. al. Phys. Rev. 56, 4960 (1997); P. Salières et. al. Adv. Atom. Molecular and Opt. Phys. 41 (1998); J.-F. Hergott et. al. Phys. Rev. A 66, 21801 (2002); A. L'Huillier et. al. Eur. Phys. J. D 26, 91 (2003); S. Kazamias et. al. Phys. Rev. A 69, 63416 (2004)

[6] Z. Chang et. al. Phys. Rev. Lett. 79, 2967 (1997); Ch. Spielmann et. al. Science 278, 661 (1997)

[7] R. Kienberger et. al. Physica Scripta T110, 32 (2004); www.attoworld.de; Kienberger et. al. Science 297, 1144 (2002)

[8] G. Mourou Appl. Phys. B 65, 205 (1997); Sciences aux temps ultracourts, Académie des Sciences, RST n 9 Sept. (2000)

[9] D. Umstadter et. al. Sciences, 273, 472 (1996); D. Umstadter et. al. Phys. Rev. Lett. 76, 2073 (1996); E. Esarey et. al. Phys. Rev. Lett. 79, 2682 (1997 ); F. Amiranoff et. al. p. 61 Images de la Physique Eds CNRS (1997)

[10] Bula et. al. Phys. Rev. Lett. 76, 3116 (1996); Burke et. al. Phys. Rev. Lett. 79, 1626 (1997)

[11] www.ipgphotonics.com, Y. Jeong et. al. Opt. Express 12, 6088 (2004); Bonatiet. al. Photonics West 2005 Late Break. Develop. Session (2005); A. Tünnermann et. al. J. Phys. B: At. Mol. Opt. Phys. 38, S681 (2005)

[12] P. Yeh et. al. J. Opt. Soc. Am. B 68, 9, 1196 (1978); S. Février Electr. Lett., 39, 1240 (2003)

[13] N. A. Mortensen et. al. Opt. Lett., 28, 1879 (2003)

[14] A. Liem et. al. Proc. Photonics West, 5335-24 (2004); R. K. Kristiansen et. al. $4^{\text {a }}$ Reunion Española de Optoelectronica, OPTOEL'05; www.crystal-fibre.com

[15] a- M. H. Ober et. al. Opt. Lett., 17, 1532 (1993); b- F. Tauser et. al. Opt. Express 11, 594 (2003)

[16] a- B. Ortaç et. al. Opt. Lett. 28, 1305 (2004); b- M. H. Ober et. al. Opt. Lett., 18, 367 (1993)

[17] a- K. Tamura et. al. Appl. Phys. Lett., 67, 158 (1995); b- F. Lim et. al. Opt. Lett., 28, 660 (2003)

[18] a- M. H. Ober et. al. Opt. Lett., 20, 2303 (1995); b- F. Ilday et. al. Opt. Lett., 28, 1362 (2003)

[19] a- J. Limpert et. al. Opt. Lett., 28, 1984 (2003); b- A. Galvanauskas et. al. Opt. Lett., 26, 935 (2001)

[20] F. Druon, Sources femtosecondes pompées par diode - Lasers \& Technologies Femtosecondes Ecole d'Eté - CNRS / Porquerolles Octobre 2005

[21] Compound Semiconductor May 2006 p. 15

[22] A. Hideur et. al. Appl. Phys. Lett., 79, 3389 (2001), A. Hideur et. al. Appl. Phys. B, 74, 121 (2002)

[23] F. Ö. Ilday et. al. Opt. Lett., 28, 1365 (2003), F. Ö. Ilday et. al. Opt. Express, 11, 3550 (2003)

[24] J. R. Buckley et. al. Opt. Lett., 30, 1888 (2005)

[25] F. Ö. Ilday et. al. Phys. Rev. Lett., 92, 213902 (2004)

[26] a- L. Kuznetsova et. al. CLEO 2005 paper CtuCC7, 2005; b- L. Shah et. al. Opt. Express, 13, 4717 (2005)

[27] F. Röser et. al. Opt. Lett. 30, 2754 (2005); F. Röser et. al. Photonics West 2006

[28] B. Ortaç et. al. Cleo-Europe Munïch (2005); ibid Photonics West, Jan. (2006), Session 10, papers 6102-40 \& 6102-80

[29] Nielsen et. al. Opt. Expr. 13, 9346 (2005); T. Schreiber et. al. Opt. Lett. 31, 574 (2006); T. Schreiber et. al. Photonics West, Jan. (2006), Session 10, paper 6102-36

[30] B. Ortaç et. al. Appl. Phys. B 79, 185 (2004); B. Ortaç et. al. IEEE Phot. Techn. Lett. 16, 1274 (2004)

[31] B. Ortaç et. al. Opt. Lett. 29, 1995 (2004); B. Ortaç et. al. Appl. Phys. B 81, 507 (2005)

[32] Ph. Grelu et. al. J. Opt. Soc. B 20, 863 (2002); Ph. Grelu et. al. Opt. Express 11, 2238 (2003)

[33] H. Leblond et. al. Phys. Rev. A 65063811 (2002), A. Komarov et. al. Phys. Rev. A 71053809 (2005), A. Komarov et. al. Phys. Rev. E 72025604 (2005)

[34] a- B. Ortaç et. al. accepté à Appl. Phys. B Mai 2006; b- B. Ortaç et. al. Opt. Express 14, 6075 (2006) 
[35] a- J.-B. Lecourt et al. JNOPM 14, 427 (2005); b- J.-B. Lecourt et al. COLOQ 9 Dijon (Sept. 2005) à paraître Journ. de Phys. IV (été 2006) 2 papiers, c- J.-B. Lecourt et al. JNOG (Nov. 2005)

[36] Y.-W. Song et. al. IEEE Photon. Techn. Lett. 171623 (2005), S. Yamashita et. al. ibid 17750 (2005). S. Set et. al. JLT 22, (2004), C. S. Goh et. al., ASSL 2005, paper CThG2

[37] G. P. Agrawal, "Nonlinear fiber optics" (1995), T. Chartier et. al. Opt. Express 11, 2561 (2003)

[38] A. Paul et. al. Nature 421, 51 (2003); E. A. Gibson et. al. Science 302, 95 (2003); H. C. Kapteyn et. al. Physics Today, 39 March 2005 